\title{
Long-term health-related quality of life after curative treatment for prostate cancer: A regional cross-sectional comparison of two standard treatment modalities
}

\author{
MARIE HJÄLM-ERIKSSON ${ }^{1,2}$, BO LENNERNÄS ${ }^{3}$, ANDERS ULLÉN ${ }^{1,2}$, \\ HEMMING JOHANSSON $^{1}$, JONAS HUGOSSON ${ }^{4}$, STEN NILSSON ${ }^{1,2}$ and YVONNE BRANDBERG ${ }^{1}$ \\ ${ }^{1}$ Department of Oncology-Pathology, Karolinska Institutet, Stockholm; ${ }^{2}$ Department of Oncology, \\ Karolinska University Hospital, Stockholm; ${ }^{3}$ Jubileumskliniken, Sahlgrenska University Hospital, Gothenburg; \\ ${ }^{4}$ Department of Urology, Sahlgrenska University Hospital, Gothenburg, Sweden
}

Received August 6, 2014; Accepted September 10, 2014

DOI: 10.3892/ijo.2014.2734

\begin{abstract}
Effects on long-term health-related quality of life (HRQoL) were evaluated in patients treated for localized prostate cancer by two standard modalities: radical retropubic prostatectomy (RP) and external beam radiotherapy combined with a high-dose-rate brachytherapy boost (HDRBT-EBRT). The HRQoL data were compared with age-adjusted normative data. Men diagnosed with localized prostate cancer and treated with curative intent in Gothenburg, Sweden, 1988-1997 were included. HRQoL was measured in October 2000 using the EORTC QLQ-C30 and EORTC QLQ-PR25 questionnaires, with a response rate of $82 \%(n=347)$. No differences in patient characteristics were found between the two treatment groups, except regarding tumor stage and PSA recurrence at the time of the questionnaires. In the RP group, $42 \%$ had T1 and 6\% had T3-4 tumors; corresponding proportions in the HDRBTEBRT group were $29 \%$ and $13 \%(\mathrm{P}=0.01)$. PSA recurrence was detected in $44 \%$ of RP patients and $9 \%$ of HDRBT-EBRT patients. In most domains, mean HRQoL scores were high and similar to the scores for the age-adjusted normative sample. However, patients reported better role and physical function compared to the normal population. We also observed more sleeping disturbances but less pain among patients than in the normal population. The disease-specific questionnaires showed statistically significant higher levels of bowel and urinary problems in the irradiated group than in the RP group, and the absolute difference between the groups was small and had minor clinical significance. We conclude that overall the general quality of life was rated high by the patients irrespective of curative treatment modality and in agreement with
\end{abstract}

Correspondence to: Dr Marie Hjälm-Eriksson, Department of Oncology, Karolinska University Hospital, SE-171 76 Stockholm, Sweden

E-mail: marie.hjalm-eriksson@karolinska.se

Key words: prostate cancer, health-related quality of life, prostatectomy, external beam radiotherapy, HDR-brachytherapy age-adjusted normative data. Statistically significant differences in bowel and urinary symptoms were found between the two treatment groups in favor of the RP group, but the clinical significance was small.

\section{Introduction}

Prostate cancer (PC) is a major health problem in men in the Western world. Definitive treatment options for localized PC routinely include radical retropubic prostatectomy (RP), external beam radiotherapy (EBRT), and brachytherapy or a combination of these methods. A recent review of 18,000 studies compared the outcomes of curative treatment of localized PC in the post-PSA era (1), and it was concluded that randomized studies are rare and there is a lack of evidence to determine whether any of the available treatment options are superior for prolonging survival. Therefore, it is necessary for patients to consider the morbidity and side-effects of the treatment modalities when deciding on treatment for PC. This underlines the importance of having detailed knowledge of health-related quality of life (HRQoL) and adverse events associated with different curative treatments. Early HRQoL research concerning treatment-related side-effects such as incontinence, bowel disturbances, and impact on sexual activity showed disparate profiles for the various treatment modalities (2-4). In the cited investigations, patients treated with RP reported more incontinence, which was acute but improved over the first 2 years after treatment. By comparison, bowel problems were worse in irradiated patients, continuing for up to 4 years, and urinary bother was increased in patients treated by brachytherapy with permanent implants. HRQoL studies concerning sexual problems have reported fewer initial problems after brachytherapy and EBRT than after RP, but the results are often hampered by a lack of baseline data.

During the 1990s, questions were raised about whether the traditional EBRT treatment dose of 66-70 Gy was sufficient to cure PC. Since then, several studies have shown increased disease-free survival after dose escalation (5). Such amplification can be done by several different methods: threedimensional conformal radiotherapy, intensity-modulated 
radiotherapy and combinations of these approaches with high-dose-rate brachytherapy (HDRBT), low-dose-rate brachytherapy or particle beam boosts. Some studies have compared acute effects of traditional and modern doseescalated radiotherapy on HRQoL in PC patients $(6,7)$, and the results indicated comparable HRQoL outcomes. However, few investigations have examined long-term effects in this clinical context.

The aim of the present study was to compare long-term HRQoL in men who were diagnosed with PC between 1988 and 1997 and had undergone one of the two curative treatment strategies that, with small adjustments, are still in routine use today. Our focus was on HRQoL after external beam radiotherapy combined with high-dose-rate brachytherapy (HDRBT-EBRT) and open retropubic prostatectomy (RP).

\section{Patients and methods}

Patients. The study cohort comprised all men who had received curative-intent treatment with either RP or HDRBTEBRT in the Gothenburg area from 1 January 1988 to 31 December $1997(n=492)$. All members of this cohort who were still alive in October 2000 were asked to participate in the HRQoL study. Two urological departments and one oncological department were involved. Evaluated patient records and death certificates covered the period 1999-2001, during which time three standard treatments [RP, EBRT and EBRT combined with HDRBT Ir-192 (radionuclide, iridium192)] and one experimental treatment [cryoablation surgery (Cryo)] were available. Men treated with EBRT alone or Cryo were not included in the study: the former because they had been treated with an old technique and inadequate doses according to the present standard; the latter because only a small selected group of patients under surveillance received Cryo treatment.

Clinical staging. TNM stage was defined according to the UICC 1992 classification (8). T stage data were obtained from patient records or from pathology reports. Patients were divided into low-, intermediate- and high-risk PC groups. The low- and high-risk groups were, respectively, classified according to the following criteria: prostate-specific antigen (PSA) $<10$ and WHO grade 1 (corresponding to Gleason score $\leq 5$ ) and T1; PSA $\geq 20$ and/or WHO grade 3 (corresponding to Gleason score $\geq 4+3$ ) and/or T3. The intermediate-risk group comprised all patients that were not included in either the lowor the high-risk group. In the high-risk PC group, lymph node dissection was performed before radiotherapy or during RP, and $\mathbf{M}$ stage was assessed by bone scan.

Radical retropubic prostatectomy (the RP group). RP was performed as an open retropubic procedure, if possible using the nerve-sparing technique. The majority of patients underwent regional lymph node excision with frozen section, and only men with N0 were included (9).

Brachytherapy combined with external beam radiotherapy (the HDRBT-EBRT group). The radiotherapy technique used has been previously described in detail (10). Briefly, the prescribed target dose was $50 \mathrm{~Gy}$, which was given in 2-Gy fractions using high-energy photons delivered by a standard four-field box technique to the prostate and seminal vesicles. The HDRBT target dose was 20 Gy in two 10-Gy fractions, which were delivered 2 weeks apart to the prostate gland and the base of the vesicles with a $3-\mathrm{mm}$ margin.

Neoadjuvant hormonal therapy (NHT). In addition to the treatment modalities described above, NHT using a GNRH analogue was given 3-6 months before start of treatment to patients with high-risk features. For irradiated patients, NHT was administered throughout the course of radiotherapy. NHT was also given to $51 \%$ of the patients in the RP group who were participating in a clinical study (11).

Follow-up. A majority of the patients underwent annual follow-up at the Department of Oncology or Urology at Sahlgrenska University Hospital, including clinical examination and measurement of PSA. A bone scan was performed if PSA relapse occurred or was suspected on clinical grounds. Most patients with relapse of disease were treated with early hormone therapy or were given the best supportive care, as recommended by the treating physician.

Data collection. After a mean follow-up time of 7 years (range, 4-16 years) in October 2000 to April 2001, all men who were still alive were asked by mail to participate in the HRQoL assessment by completing a HRQoL questionnaire and returning it in a prepaid envelope. The Regional Cancer Registry in Gothenburg handled all questionnaires and entered data in the study database. One reminder was sent.

Instruments. The European Organization of Research and Treatment of Cancer (EORTC) developed the Quality of Life Questionnaire C30 (EORTC QLQ-C30) to measure HRQoL in cancer patients participating in clinical trials (12). This instrument includes 30 items comprising five functional scales (physical, role, emotional, social and cognitive), three symptom scales (fatigue, pain, nausea and vomiting), a global health status/QoL scale, and six single items (dyspnea, loss of appetite, insomnia, constipation, diarrhea and financial impact of disease). In addition to the EORTC QLQ-C30, we used the EORTC QLQ-PR25 (13), which is a disease-specific questionnaire assessing problems related to treatment of PC by use of 25 questions on areas such as sexual function and bladder and bowel problems. During the period covered by the present study, the EORTC QLQ-PR25 had not yet been validated.

Statistical methods. The items of the EORTC QLQ-C30 and QLQ-PR25 instruments were scaled according to the scoring manual (14). Raw scores were transformed into a scale ranging from 0 to 100 . Higher scores indicate better functioning on the functional subscales and the global quality of life scale, and more symptoms on the symptom scales. The expected mean value for each of the EORTC QLQ-C30 subscales was calculated using the age distribution in all groups combined with age-specific mean reference scores from the Swedish population (15). Differences in categorical variables were tested using Fisher's exact test. Continuous variables were modeled using linear regression. Group differences were assessed by Wald tests. Results from the regression models are presented as mean differences together with $99 \%$ confidence intervals. 
Table I. Number of deaths before questionnaires, and number and proportions of respondents per treatment group.

\begin{tabular}{lcccr}
\hline Treatment group & Initial cohort $\mathrm{n}$ & $\begin{array}{c}\text { Deceased before } \\
\text { questionnaires, } \mathrm{n}(\%)\end{array}$ & $\begin{array}{c}\text { Questionnaires } \\
\text { sent, } \mathrm{n}(\%)\end{array}$ & Respondents, $\mathrm{n}(\%)$ \\
\hline RP & 379 & $48(13)$ & $331(79)$ & $261(79)$ \\
HDRBT/EBRT & 113 & $23(20)$ & $90(21)$ & $86(96)$ \\
Total & 492 & $71(14)$ & 421 & $347(82)$ \\
\hline
\end{tabular}

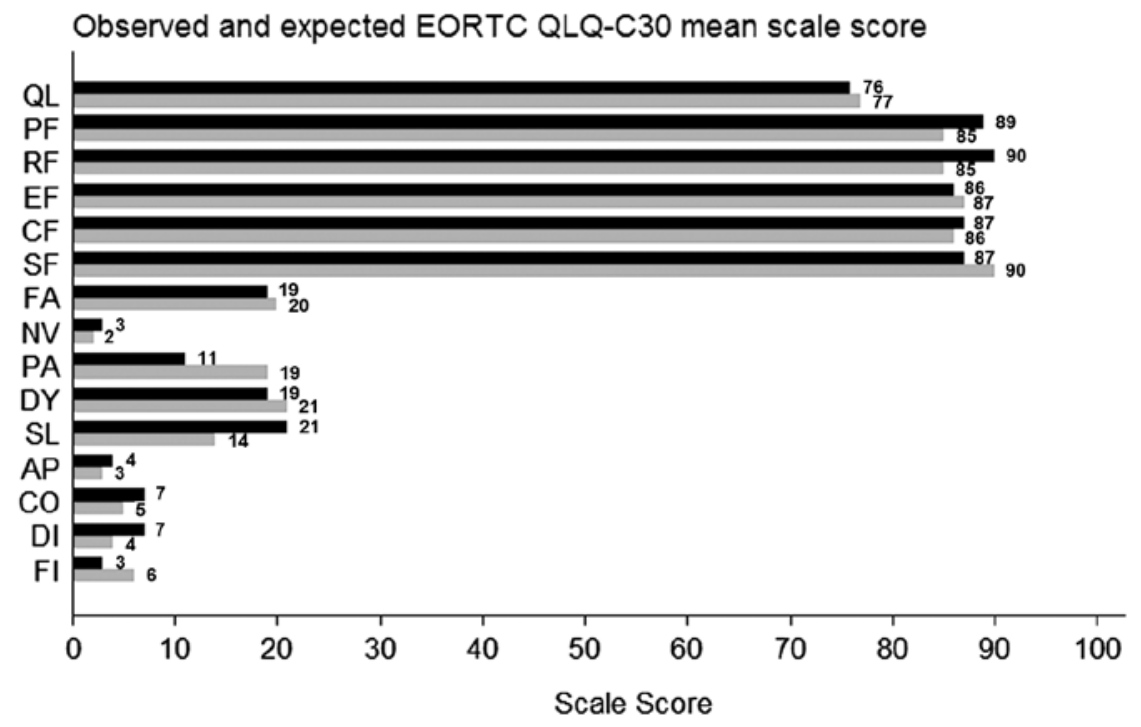

Observed

Expected

Figure 1. Comparison between HRQoL, mean scores in the study population (observed) and an age-adjusted normative sample (expected). Functional scales (high scores=high levels of functioning): QL, global quality of life; PF, physical function; RF, role function; EF, emotional function; CF, cognitive function; SF, social function. Symptom scales (high scores=high levels of sypmtoms); FA, fatigue; NV, nausea; PA, pain; DY, dyspnea; SL, sleeping disturbances; AP, appetite loss; CO, constipation; DI, diarrhea; FI, financial difficulties.

In the interpretation of the QLQ-C30 scores, a difference of $\geq 5$ points on the 0 - 100 scale was considered clinically significant. Differences of 5-9 points were considered small, 10-20 as moderate and $>20$ as large (16). Due to multiple testing, the level of significance was set at 0.01 . All statistical analyses were performed using the Stata statistical software version 11.

\section{Results}

The initial cohort comprised 492 patients, but 71 (14.4\%) of those individuals died before onset of the study. Thus, 421 patients were asked to participate in the HRQoL evaluation; $347(82 \%)$ completed the questionnaires, and 42 declined to take part or did not respond. The initial number of patients in each treatment group, the number of respondents, and the number of deaths before administration of the questionnaires are listed in Table I.

Clinical and demographic patient characteristics for each treatment group are presented in Table II. There were no statistically significant differences in clinical parameters between the groups, except regarding $\mathrm{T}$ stage and PSA recurrence at the time of the questionnaires. The proportion of patients with locally advanced disease (T3-T4 tumors) was larger in the HDRBT-EBRT group than in the RP group (13 and 6\%, respectively; $\mathrm{P}=0.01)$. PSA relapse at the time of the questionnaires was noted in $44 \%$ of the men in the RP group compared to $9 \%$ in the HDRBT-EBRT group $(\mathrm{P}<0.0001)$. All demographic parameters except education were equally distributed between the treatment groups.

HRQoL results for the normative population and the treatment groups are shown in Fig. 1. In general, the patients' mean HRQoL scores were high and similar to the mean scores for the normative sample, with the exception of physical and role function, for which the patients' scores were higher. Considering physical symptoms, compared to the normal population, the patients reported less pain but more pronounced problems with sleep disturbances.

The results of the univariate and multivariate analyses comparing the two treatment groups are outlined in Table III. Taking into account age, PSA recurrence, and neo-adjuvant hormonal treatment, there were no statistically significant differences between the groups in either the univariate or the multivariate regression analysis of the functional domains in the EORTC QLQ C-30 questionnaire. Adding 'risk group at diagnosis' or 'time from diagnosis to time for questionnaire' as confounding factors did not change the results (data not shown). Concerning the symptom scale, a higher level of problem with diarrhea was found in the HDRBT-EBRT group, 
Table II. Clinical and demographic characteristics of the patients in the two treatment groups.

\begin{tabular}{|c|c|c|c|c|}
\hline & Prostatectomy & HDRBT-EBRT & P-value & Total \\
\hline No. of patients (\%) & $261(75)$ & $86(25)$ & & $347(100)$ \\
\hline \multicolumn{5}{|l|}{ Age (years) } \\
\hline Mean (SD) & $70(6.0)$ & $70(6.2)$ & \multirow{2}{*}{$N S^{f}$} & $70(6.1)$ \\
\hline Median (range) & $70(51-83)$ & $70(56-83)$ & & $70(51-83)$ \\
\hline \multicolumn{5}{|l|}{ WHO, n (\%) } \\
\hline 1 & $108(41)$ & $33(38)$ & \multirow{4}{*}{ NS } & $141(41)$ \\
\hline 2 & $113(43)$ & $35(41)$ & & $148(43)$ \\
\hline 3 & $32(12)$ & $4(5)$ & & $36(10)$ \\
\hline Missing & $8(3)$ & $14(16)$ & & $22(6)$ \\
\hline \multicolumn{5}{|l|}{ Clinical T stage, $\mathrm{n}(\%)$} \\
\hline 1 & $111(42)$ & $25(29)$ & \multirow{5}{*}{0.01} & $136(39)$ \\
\hline 2 & $132(51)$ & $49(57)$ & & $181(52)$ \\
\hline 3 & $16(6)$ & $11(13)$ & & $27(8)$ \\
\hline 4 & $0(0)$ & $1(1)$ & & $1(0)$ \\
\hline Missing & $2(1)$ & 0 & & $2(1)$ \\
\hline \multicolumn{5}{|l|}{ PSA (ng/ml) } \\
\hline Mean & $16(29.9)$ & $12(9.1)$ & \multirow{2}{*}{$N S^{f}$} & $15.3(26.4)$ \\
\hline Median (range) & $9.2(0.9-410)$ & $9.6(0.5-36)$ & & $9.4(0.5-410)$ \\
\hline \multicolumn{5}{|l|}{ Risk group, ${ }^{\mathrm{a}} \mathrm{n}(\%)$} \\
\hline Low & $37(14)$ & $5(6)$ & \multirow{4}{*}{ NS } & $42(12)$ \\
\hline Intermediate & $123(47)$ & $39(45)$ & & $162(47)$ \\
\hline High & $92(35)$ & $30(35)$ & & $122(35)$ \\
\hline Missing & $9(3)$ & $12(14)$ & & $21(6)$ \\
\hline Neoadjuvant hormonal therapy, $\mathrm{n}(\%)$ & $133(51)$ & $49(57)$ & NS & $182(52)$ \\
\hline Relapse at time of questionnaires, $\mathrm{n}(\%)$ & $114(44)$ & $8(9)$ & $<0.000$ & $122(35)$ \\
\hline \multicolumn{5}{|l|}{ Civil status, $\mathrm{n}(\%)$} \\
\hline Married & $215(82)$ & $73(85)$ & \multirow{5}{*}{ NS } & $288(83)$ \\
\hline Single & $20(8)$ & $8(9)$ & & $28(8)$ \\
\hline Single with partner & $14(5)$ & $4(5)$ & & $18(5)$ \\
\hline Widower & $11(4)$ & $1(1)$ & & $12(3)$ \\
\hline Missing & $1(0)$ & & & $1(0)$ \\
\hline \multicolumn{5}{|l|}{ Employment, n (\%) } \\
\hline Gainfully employed & $29(11)$ & $13(15)$ & & $42(12)$ \\
\hline Retired (age >65) & $217(83)$ & $66(77)$ & & $283(82)$ \\
\hline On sick leave ${ }^{\mathrm{b}}$ & $11(4)$ & $6(7)$ & & $17(5)$ \\
\hline Other & $3^{c}(1)$ & $1^{\mathrm{d}}(1)$ & & $4(1)$ \\
\hline Missing & $1(0)$ & $1(1)$ & NS & $2(1)$ \\
\hline \multicolumn{5}{|l|}{ Education, n (\%) } \\
\hline Comprehensive school & $162(62)$ & $42(49)$ & & 204 (59) \\
\hline Higher school degree & $23(9)$ & $7(8)$ & & $30(9)$ \\
\hline University degree & $74(28)$ & $37(43)$ & 0.007 & $111(32)$ \\
\hline Missing & $2(1)$ & & & $2(1)$ \\
\hline \multicolumn{5}{|l|}{ Nationality, n (\%) } \\
\hline Swedish & $232(89)$ & $77(90)$ & & $309(89)$ \\
\hline Scandinavian & $10(4)$ & $4(5)$ & & $14(4)$ \\
\hline European (other than above) & $15(6)$ & $4(5)$ & & $19(5)$ \\
\hline Non-European & $3(1)$ & $1(1)$ & NS & $4(1)$ \\
\hline Missing & $1(0)$ & & & $1(0)$ \\
\hline
\end{tabular}

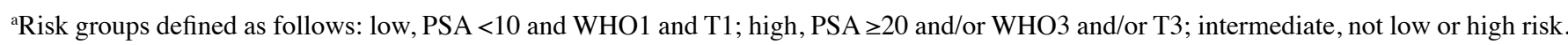

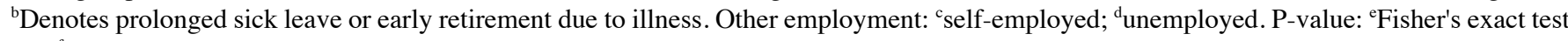
and ${ }^{\mathrm{f}} \mathrm{F}$-test for continuous variables. 
Table III. Univariate and multivariate analyses of HRQoL subscales and single items, taking into account age, recurrence of PC at time of questionnaires, and neoadjuvant hormonal treatment.

\begin{tabular}{|c|c|c|c|c|c|c|c|}
\hline \multirow[b]{2}{*}{ Subscale } & \multicolumn{4}{|c|}{ Univariate analysis } & \multicolumn{3}{|c|}{ Multivariate analysis } \\
\hline & Mean (SD) & Mean difference & $99 \% \mathrm{CI}$ & P-value & Mean difference & $99 \% \mathrm{CI}$ & P-value \\
\hline \multicolumn{8}{|c|}{ Global quality of life $\mathrm{e}^{\mathrm{a}}$} \\
\hline HDRBT-EBRT & $74(20)$ & -3 & $(-10$ to 4$)$ & & $-6^{c}$ & $(-14$ to 2$)$ & \\
\hline $\mathrm{RP}$ & $77(22)$ & ref & & 0.245 & ref & & 0.053 \\
\hline \multicolumn{8}{|l|}{ Physical function $^{\mathrm{a}}$} \\
\hline HDRBT-EBRT & $88(16)$ & -1 & $(-6$ to 4$)$ & & -3 & $(-8$ to 2$)$ & \\
\hline $\mathrm{RP}$ & $89(16)$ & ref & & 0.61 & ref & & 0.219 \\
\hline \multicolumn{8}{|l|}{ Role function $^{\mathrm{a}}$} \\
\hline HDRBT-EBRT & $89(22)$ & -2 & $(-9$ to 5$)$ & & $-5^{c}$ & $(-12$ to 3$)$ & \\
\hline $\mathrm{RP}$ & $91(21)$ & ref & & 0.448 & ref & & 0.094 \\
\hline \multicolumn{8}{|c|}{ Emotional function $^{\mathrm{a}}$} \\
\hline HDRBT-EBRT & $86(19)$ & -0.3 & $(-6$ to 6$)$ & & -2 & $(-8$ to 5$)$ & \\
\hline $\mathrm{RP}$ & $86(19)$ & ref & & 0.893 & ref & & 0.459 \\
\hline \multicolumn{8}{|c|}{ Cognitive function $^{\mathrm{a}}$} \\
\hline HDRBT-EBRT & $85(15)$ & -3 & $(-8$ to 2$)$ & & -4 & $(-9$ to 1$)$ & \\
\hline $\mathrm{RP}$ & $87(16)$ & ref & & 0.168 & ref & & 0.06 \\
\hline \multicolumn{8}{|l|}{ Social function $^{\mathrm{a}}$} \\
\hline HDRBT-EBRT & $85(20)$ & -2 & $(-9$ to 5$)$ & & -4 & $(-11$ to 4$)$ & \\
\hline $\mathrm{RP}$ & $87(22)$ & ref & & 0.454 & ref & & 0.224 \\
\hline \multicolumn{8}{|l|}{ Fatigue $^{\mathrm{b}}$} \\
\hline HDRBT-EBRT & $20(19)$ & 2 & $(-5$ to 9$)$ & & $5^{c}$ & $(-3$ to 12$)$ & \\
\hline $\mathrm{RP}$ & $18(22)$ & ref & & 0.446 & ref & & 0.112 \\
\hline \multicolumn{8}{|l|}{ Nausea/vomiting ${ }^{\mathrm{b}}$} \\
\hline HDRBT-EBRT & $3(7)$ & -0.4 & -3 to 3 & & 0.6 & -3 to 4 & \\
\hline $\mathrm{RP}$ & $3(10)$ & & & 0.729 & & & 0.615 \\
\hline \multicolumn{8}{|l|}{ Pain $^{\mathrm{b}}$} \\
\hline HDRBT-EBRT & $12(21)$ & 2 & $(-6$ to 9$)$ & & 3 & $(-5$ to 11$)$ & \\
\hline $\mathrm{RP}$ & $10(23)$ & ref & & 0.567 & ref & & 0.357 \\
\hline \multicolumn{8}{|l|}{ Dyspnea $^{\mathrm{b}}$} \\
\hline HDRBT-EBRT & $20(24)$ & 0.7 & $(-8$ to 9$)$ & & $6^{\mathrm{c}}$ & $(-3$ to 15$)$ & \\
\hline $\mathrm{RP}$ & $19(27)$ & ref & & 0.832 & ref & & 0.108 \\
\hline \multicolumn{8}{|l|}{ Insomnia ${ }^{b}$} \\
\hline HDRBT-EBRT & $23(26)$ & 3 & $(-6$ to 12$)$ & & $6^{3}$ & $(-4$ to 15$)$ & \\
\hline $\mathrm{RP}$ & $20(28)$ & ref & & 0.413 & ref & & 0.121 \\
\hline \multicolumn{8}{|l|}{ Appetite loss ${ }^{\mathrm{b}}$} \\
\hline HDRBT-EBRT & $4(10)$ & -0.3 & $(-5$ to 4$)$ & & 1 & $(-4$ to 6$)$ & \\
\hline $\mathrm{RP}$ & $4(14)$ & ref & & 0.870 & ref & & 0.556 \\
\hline \multicolumn{8}{|l|}{ Constipation $^{\mathrm{b}}$} \\
\hline HDRBT-EBRT & $7(18)$ & 0.02 & $(-6$ to 6$)$ & & 2 & $(-5$ to 9$)$ & \\
\hline $\mathrm{RP}$ & 7 (19) & ref & & 0.032 & ref & & 0.443 \\
\hline \multicolumn{8}{|l|}{ Diarrhea $^{\mathrm{b}}$} \\
\hline HDRBT-EBRT & $10(19)$ & 5 & $(-0.05$ to 10$)$ & & $7^{c}$ & (1 to 12$)$ & \\
\hline $\mathrm{RP}$ & $5(15)$ & ref & & 0.011 & ref & & $<0.002$ \\
\hline
\end{tabular}

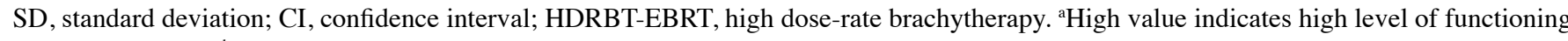
and quality of life. ${ }^{b}$ High value indicates high level of symptom and problems. ${ }^{c}$ Small clinical difference. 
Table IV. Prostate cancer specific problems QLQ-PR25: univariate and multivariate analyses of HRQoL subscales EORTC QLQ-PR25, taking into account age, recurrence of PC at time of questionnaires and neoadjuvant hormonal treatment.

\begin{tabular}{|c|c|c|c|c|c|c|c|}
\hline \multirow[b]{2}{*}{ Subscale } & \multicolumn{4}{|c|}{ Univariate analysis } & \multicolumn{3}{|c|}{ Multivariate analysis } \\
\hline & Mean (SD) & Mean difference & $99 \%$ CI & P-value & Mean difference & $99 \%$ CI & P-value \\
\hline \multicolumn{8}{|l|}{ Urinary function ${ }^{\mathrm{a}}$} \\
\hline HDRBT-EBRT & $19(17)$ & 4 & $(-1$ to 9$)$ & & $6^{\mathrm{b}}$ & (0.2 to 11$)$ & \\
\hline $\mathrm{RP}$ & $15(16)$ & ref & & $<0.046$ & ref & & 0.008 \\
\hline \multicolumn{8}{|l|}{ Bowel function ${ }^{a}$} \\
\hline HDRBT-EBRT & $9(12)$ & 5 & (0.7 to 9$)$ & & $5^{\mathrm{b}}$ & (1 to 10 ) & \\
\hline $\mathrm{RP}$ & $4(9)$ & ref & & 0.003 & ref & & 0.001 \\
\hline \multicolumn{8}{|l|}{ Sexual activity ${ }^{\mathrm{a}}$} \\
\hline HDRBT-EBRT & $30(27)$ & -3 & $(-13$ to 7$)$ & & -3 & $(-13$ to 7$)$ & \\
\hline $\mathrm{RP}$ & $33(30)$ & ref & & 0.408 & ref & & 0.431 \\
\hline \multicolumn{8}{|l|}{ Sexual function } \\
\hline HDRBT-EBRT & $60(21)$ & 4 & (-11 to 18$)$ & & -4 & $(-12$ to 20$)$ & \\
\hline $\mathrm{RP}$ & $56(27)$ & ref & & 0.528 & ref & & 0.546 \\
\hline \multicolumn{8}{|c|}{$\begin{array}{l}\text { Hormone treatment-related } \\
\text { symptoms }{ }^{\mathrm{a}}\end{array}$} \\
\hline HDRBT-EBRT & $12(10)$ & -0.9 & (-5 to 3$)$ & & 1 & (-4 to 5$)$ & \\
\hline $\mathrm{RP}$ & $13(13)$ & ref & & 0.557 & ref & & 0.616 \\
\hline \multicolumn{8}{|l|}{ Incontinence aid ${ }^{a}$} \\
\hline HDRBT-EBRT & $27(38)$ & 11 & $(-13$ to 35$)$ & & $7^{\mathrm{b}}$ & (-19 to 33$)$ & \\
\hline RP & $16(25)$ & ref & & 0.256 & ref & & 0.491 \\
\hline
\end{tabular}

EORTC QLQ-PR25 item 20

Sexually active HDRBT-EBRT

RP P-value

Yes, $\mathrm{n}(\%)$

$32(37)$

$96(37)$

No, n $(\%)$

$54(63)$

$165(63)$

0.521

SD, standard deviation; CI, confidence interval; HDRBT-EBRT, high dose-rate brachytherapy + external beam radiation therapy; RP, radical

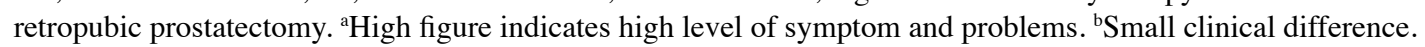

and this difference was both statistically and clinically significant. Small clinically significant differences favoring the RP group were found for global quality of life, role functioning, fatigue, dyspnea, insomnia and diarrhea, but none of these differences were statistically significant.

Results concerning the disease-specific and treatmentrelated symptoms are presented in Table IV. Levels of bowel-related symptoms and urinary problems were higher in the HDRBT-EBRT patients than in the RP patients, and these results were statistically significant but the clinical significance were small. There was no statistically significant difference in sexual activity between the two treatment groups, although only $37 \%$ of the patients reported that they were sexually active and sexual function was low (mean score 57, SD 25).

\section{Discussion}

Clinically localized PC can be treated effectively by use of conceptually different treatment approaches with contrasting patterns of acute and late side-effects. Hence overall and disease-specific HRQoL following treatment has become an important aspect in patients with such disease. In general, the studies published to date concern HRQoL during the first 3-5 years after treatment. The number of longer follow-ups is limited, and, in particular, few investigations have compared late effects of surgery and modern dose-escalated radiotherapy.

The present cross-sectional cohort study explored longterm HRQoL in PC patients 7 years after curative treatment by two different methods, RP and HDRBT-EBRT. The results were compared with age-matched HRQoL data on the normal male population in Sweden. We found that the levels of overall quality of life were high, irrespective of curative treatment modality. In short, the scores were high on functional scales and low on symptom scales in all domains, and they concurred with age-adjusted normative data. The data from the diseasespecific questionnaire revealed a small but statistically significant difference in bowel and urinary problems between the treatment groups, in favor of the RP group.

The differences we observed between the study groups agree with the findings of other investigators concerning 
general HRQoL in PC patients following curative treatment (17-20). In the disease-specific EORTC QLQ-PR25 instrument, more bowel and urinary tract symptoms were reported by patients in the HDRBT-EBRT group than by those in the RP group. Similar results concerning bowel symptoms have been reported by other researchers $(3,17-20)$. These studies also revealed a pattern of increased urinary problems and incontinence in RP patients, but more extensive urinary bother (e.g., urgency and frequency) among irradiated patients, especially those treated with permanent seeds. HRQoL data on the combined HDRBT-EBRT treatment are limited. However, similar to our findings, a study of HRQoL 5 years after treatment in a cohort of PC patients given HDR brachytherapy and EBRT indicated that urinary symptoms were more common than bowel symptoms (21). The authors suggested that the standard strategy applied at the time of their investigation (i.e., assuming that a central position of the urethra represents a good estimate of the location of urethra, when defining the treatment dose) might have led to administration of higher doses to the urethra than intended and hence affected the incidence of treatment-induced chronic urethritis. A similar technique was used in Gothenburg in the 1990s, which might explain the difference observed in the present study. Moreover, other investigators (19) have reported that urinary and bowel symptoms, as well as sexual problems, are increased after radiotherapy combined with NHT.

Only about one third of the patients in our study were sexually active, and no differences in sexual function were found between the treatment groups. The interpretation of the results in this domain was limited by lack of individual baseline data and data on the normal population. Our analyses were also restricted by the small sample size with respect to this variable. In a comparison of men treated with EBRT, permanent seed implants, and RP, Litwin et al (18) noted that the EBRT group reported superior sexual function after 24 months compared to the other groups. However, severe sexual problems were experienced to the same extent in all groups, regardless of treatment. Other studies of HRQoL have demonstrated a general decrease in sexual function after 3-5 years of follow-up in patients treated with RP and EBRT (2019).

The differences we observed between the patient cohort and the normal population agree with the results of a 5-year follow-up investigation of HRQoL in Swedish PC patients (20), which showed that the patients had better physical and role function and lower levels of pain compared to men in the normal population. An explanation for this might be that the PC patients based their questionnaire responses on different standards compared to the non-PC subjects, a phenomenon that has been referred to as 'response shift' (22).

Limitations of the present study include the lack of baseline HRQoL assessment and the lack of randomization to treatment, thus, it is possible that confounding factors affected the results. Although, the RP and the HDRBT-EBRT groups were equivalent regarding the majority of clinical parameters previously shown to be relevant, there was a striking difference between the two groups concerning the PSA recurrence rate at the time of the questionnaires (44 and 9\%, respectively). A plausible explanation for this discrepancy is that different definitions of PSA recurrence were applied in our study depending on the treatment modality used: for RP, PSA $\geq 0.2 \mathrm{ng} / \mathrm{ml}$; for
HDR-EBRT, PSA $\geq 2 \mathrm{ng} / \mathrm{ml}$ above nadir. The present study was performed in the early 2000s, at which time RP and doseescalated radiotherapy were the standard treatment options for localized PC. Technically, both the open RP and the HDRBTEBRT are still performed in manners similar to those that were employed during the study period. Therefore, our results can provide physicians and PC patients with valuable information concerning long-term effects on HRQoL after treatment of PC, which is particularly important considering that few investigations have compared long-term HRQoL after RP and dose-escalated radiotherapy.

The present study also had several strengths. First of all, it was large, population based, and had a high overall response rate $(82 \%)$. Furthermore, the public health system in Sweden offers a unique opportunity to follow patients over long periods, and thus, potential inter-individual differences in staging, grading, treatment procedures, and follow-up during the study period were at a minimum in our investigation. Another advantage is that two validated questionnaires were used in our evaluation, one of which is disease specific, and age-adjusted normative data were available.

In conclusion, we found that long-term HRQoL after curative treatment of localized PC by RP or HDRBT-EBRT was high and agreed with age-adjusted normative data. Statistically significant differences in bowel and urinary symptoms were observed in favor of the RP group, but the clinical significance concerning these disparities was small.

\section{Acknowledgements}

The present study was funded by the Swedish Cancer Society, Radiumhemmets Research Foundation, and Af Jocknick Research Foundation. Grants for data collection were provided by the Jubilee Clinical Research Foundation against Cancer.

\section{References}

1. Grimm P, Billiet I, Bostwick D, et al: Comparative analysis of prostate-specific antigen free survival outcomes for patients with low, intermediate and high risk prostate cancer treatment by radical therapy. Results from the Prostate Cancer Results Study Group. BJU Int 109 (Suppl 1): 22-29, 2012.

2. Frank SJ, Pisters LL, Davis J, et al: An assessment of quality of life following radical prostatectomy, high dose external beam radiation therapy and brachytherapy iodine implantation as monotherapies for localized prostate cancer. J Urol 178: 2151-2156, 2007.

3. Wei JT, Dunn RL, Sandler HM, et al: Comprehensive comparison of healthrelated quality of life after contemporary therapies for localized prostate cancer. J Clin Oncol 20: 557-566, 2002.

4. Namiki S and Arai Y: Health-related quality of life in men with localized prostate cancer (Rewiev). Int J of Urol 17: 125-138, 2010.

5. Viani GA, da Silva LG and Stefano EJ: High-dose conformal radiotherapy reduces prostate cancer-specific mortality: results of a meta-analysis. Int J Radiat Oncol Biol Phys 83: e619-e625, 2012.

6. Vordermark D, Wulf J, Markert K, et al: 3-D conformal treatment of prostatecancer to $74 \mathrm{~Gy}$ vs. high-dose-rate brachytherapy boost: a cross-sectional quality-of-life survey. Acta Oncol 45: 708-716, 2006.

7. Joseph KJ, Alvi R, Skarsgard D, et al: Analysis of health related quality of life of patients with clinically localized prostate cancer, one year after treatment with external beam radiotherapy (EBRT) alone versus EBRT and high dose rate brachytherapy. Radiat Oncol 3: 20, 2008.

8. American Joint Committee on Cancer. Manual for Staging of Cancer. 4th edition. Lippincott Raven, Philadelphia, 1992. 
9. Grenabo L, Grundtman S and Hedelin H: Laparoscopic obturator lymph node dissection in patients with prostatic cancer. Scand J Urol Nephrol 29: 51-55, 1995.

10. Lennernäs B, Rikner G, Letocha $\mathrm{H}$, et al: External beam radiotherapy of localized prostatic adenocarcinoma. Acta Oncol 34: 953-958, 1995

11. Hugosson J, Abrahamsson PA, Ahlgren G, et al: The risk of malignancy in the surgical margin at radical prostatectomy reduced almost three-fold in patients given neo-adjuvant hormone treatment. Eur Urol 29: 413-419, 1996.

12. Aaronson NK, Ahmedzai S, Bergman B, et al: The European Organization for Research and Treatment of Cancer QLQ-C30: a quality-of-life instrument for use in international clinical trials in oncology. J Natl Cancer Inst 85: 365-376, 1993.

13. Van Andel G, Bottomley A, Fosså SD, et al: An international field study of the EORTC QLQ-PR25: a questionnaire for assessing the health-related quality of life of patients with prostate cancer. Eur J Cancer 44: 2418-2424, 2008.

14. Fayers P, Aaronson N, Bjordahl K, et al: The EORTC QLQ-C30 scoring Manual. 3rd edition. European Organization for Cancer Research and Treatment of Cancer, Brussels, 2001.

15. Michelson $\mathrm{H}$, Bolund $\mathrm{C}$, Nilsson $\mathrm{B}$, et al: Health-related quality of life measured by the EORTC QLQ-C30 - reference values from a large sample of Swedish population. Acta Oncol 39: $477-484,2000$
16. Osoba D, Rodrigues G, Myles J, et al: Interpreting the significance of changes in health related quality-of-life scores. J Clin Oncol 16: 139-144, 1998.

17. Hoffman RM, Gilliland FD, Penson DF, et al: Cross-sectional and longitudinal comparisons of health-related quality of life between patients with prostate carcinoma and matched controls. Cancer 101: 2011-2019, 2004.

18. Litwin MS, Gore JL, Kwan L, et al: Quality of life after surgery, external beam irradiation, or brachytherapy for early-stage prostate cancer. Cancer 109: 2239-2247, 2007.

19. Sanda MG, Dunn RL, Michalski J, et al: Quality of life and satisfaction with outcome among prostate-cancer survivors. N Engl J Med 358: 1250-1261, 2008

20. Potosky AL, Davis WW, Hoffman RM, et al: Five-year outcomes after prostatectomy or radiotherapy for prostate cancer: the prostate cancer outcomes study. J Natl Cancer Inst 96: 1358-1367, 2004.

21. Wahlgren T, Nilsson S, Lennernäs B and Brandberg Y: Promising long-term health related quality of life after high-dose-rate brachytherapy boost for localized prostate cancer. Int J Radiat Oncol Biol Phys 69: 662-670, 2007.

22. Sprangers MAG and Schwartz CE: Integrating response shift into health-related quality of life research: a theoretical model. Soc Sci Med 48: 1507-1515, 1999. 